\title{
DEHAZING RESEARCH ON BRIGHTNESS EQUALIZATION MODEL OF DRONE IMAGE
}

\author{
SU Jun-ming ${ }^{1}$, LIU Li-long ${ }^{1,2^{*}}$,WAN Qing-tong ${ }^{1}$, YANG Yun-zhen ${ }^{1}$, LI Fen-fen ${ }^{1}$ \\ ${ }^{1}$ Guilin University of Technology, Guilin, China - gx_suesue@163.com \\ ${ }^{2}$ Guangxi Key Laboratory of Spatial Information and Geomatics, Guilin University of Technology, Guilin, China - \\ hn_liulilong@163.com
}

KEY WORDS: Digital Images Processing, Images Enhancement, Drone Image, HSI Transform, Brightness Equalization Model

\begin{abstract}
:
Due to the rapid development of drone technology, aerial imagery of drones is increasingly used in various fields. However, the aerial image of the drone is highly susceptible to weather conditions during the imaging process. Most aerial images are inevitably affected by fog when they are acquired. Due to the scattering and absorption of the atmosphere, the aerial image of the drone in foggy days has the characteristics of low contrast and unclear scenery. Due to the scattering and absorption of the atmosphere, the aerial image of drone acquired in the foggy environment has the characteristics of low contrast and unclear scenery. The Defogging technology for aerial image of drone can obtain a large amount of useful information in a pictures with low information amount through a certain image processing method, and convert the image with low information amount into a useful image. Therefore, the image processing research carried out for such image degradation caused by natural phenomena has universal practical significance. Aiming at the problem that the aerial image of drone is often affected by haze and the image is blurred and the image quality is degraded, this paper proposes a new model for defogging aerial image of drone. The brightness equalization model is used to improve the degraded image with fog defects. The brightness equalization model obtains the brightness channel of the original image based on the HSI transform. The brightness equalization filter is used to dynamically adjust the brightness to the appropriate interval to achieve the purpose of defogging and then further optimizes the result image by using Gaussian blur and color reshaping. Two images with fog problems were compared, using the brightness equalization model of this paper. And the quality evaluation parameters are selected to evaluate the processing results of the dehazing model. The average value of the images processed by the model is more suitable and the main quality evaluation parameters such as standard deviation and entropy are better than those of the original image.The experimental results show that the brightness equalization model can effectively remove the influence of fog in the aerial image of the drone and improve the visual effect of the image.
\end{abstract}

\section{INTRODUCTION}

In recent years, the emerging drones remote sensing technology has made up for the lack of flexibility and adaptability of satellite remote sensing and aerial remote sensing, and has become a powerful complement to the latter two ${ }^{[1]}$, playing an important role in resource management and emergency rescue. However, the quality of aerial imagery of drones fluctuates greatly in foggy environments ${ }^{[2]}$. In the foggy environment, atmospheric particles have scattering and refraction effects on light ${ }^{[3]}$, resulting in drone image contrast reduction, loss of detail, and lack of visual vividness ${ }^{[4]}$.The degradation of image quality has greatly restricted the validity and reliability of images ${ }^{[5]}$. Therefore, how to remove the influence of fog and improve the visual effect of images is a subject that has received extensive attention.

At present, the defogging processing methods for images are mainly divided into two categories ${ }^{[6][7]}$ : image restoration 
methods based on physical models and image enhancement methods. The image restoration methods mainly considers the root cause of the fog image degradation on the physical field, and analyzes the whole process of atmospheric scattering and its final influence on the image, and then inverts the process to obtain the color recovery image. For example, dark channel prior image defogging algorithm ${ }^{[8]}{ }^{[9]}$ and so on. The main disadvantage of such methods is that additional parameters are needed to establish foggy image degradation models, such as depth of field information, etc. However, in practical applications, users generally cannot obtain these information. The image enhancement method is to enhance the contrast and highlight the feature information to achieve the purpose of defogging. For example, homomorphic filtering image dehazing method ${ }^{[10]}$, Retinex theory ${ }^{[11]}{ }^{[12]}$ and so on.

In order to remove the interference of fog and make the image clearer and more realistic, this paper based on HSI color transform ${ }^{[13]}$ according to the spatial positional relationship of brightness. Using local adaptive filtering to reconstruct the brightness and nonlinear dynamic adjustment to achieve the purpose of defogging. After the HSI inverse transform, the Gaussian blur is used to preserve the image detail information and the color reshaping algorithm is used to maintain the

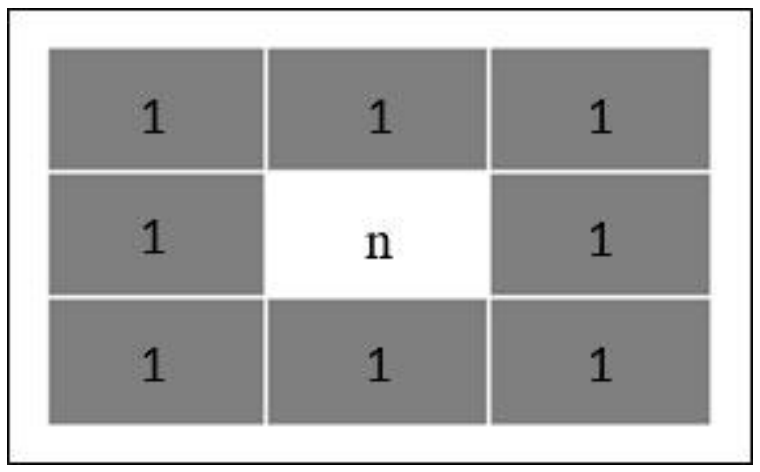

(a) Before Gaussian Blur vividness of the image color. It has been proved by experiments that the model of this paper can obviously remove the influence of foggy environment and maintain the visual effect of image detail texture, and the color of image is basically free from distortion.

\section{HSI COLOR SPACE}

In image processing, there are usually two kinds of color space: RGB color space composed of three primary colors of red, green, and blue, and the other is HSI color space.The HSI color space consists of three variables: hue (category of color), saturation (purity of color), and Intensity (lightness and darkness perceived by the human eye).

The HSI color space represents images in terms of hue, saturation and brightness [14]. Since the human eye is very sensitive to changes in brightness, the use of HSI color space is in line with human visual characteristics.

\section{GAUSSIAN BLUR}

Gaussian blur is an image processing algorithm that combines Gaussian distribution and convolution filtering, as shown in the following figure:

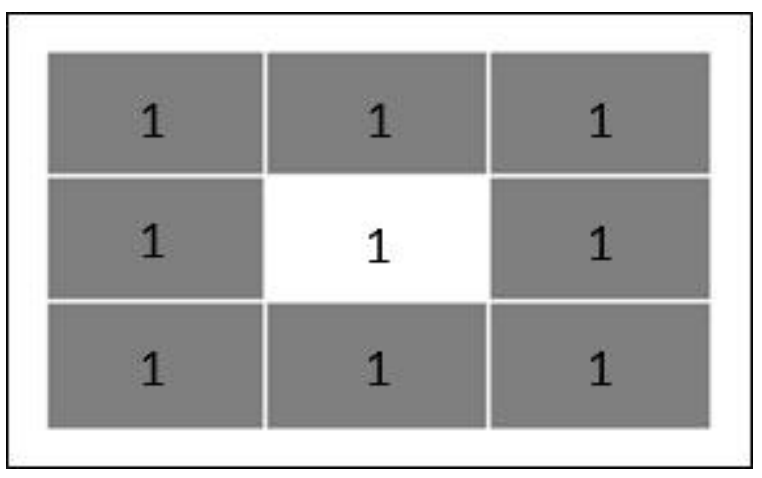

(b) After Gaussian Blur

Fig.1 Schematic diagram of Gaussian blur

The value of the central pixel point $\mathrm{n}$ is equal to the average of the neighboring pixel points. Obviously the pixel value at the center of the above image should be 1 . This $G(x, y)=\frac{1}{2 \pi \sigma^{2}} e^{-\frac{x^{2}+y^{2}}{2 \sigma^{2}}}$ preserves subtle changes in the image texture on the visual display of the image.

where

$$
\begin{aligned}
& G(x, y)=\text { weigh } \\
& \sigma=\text { variance } \\
& (x, y)=\text { image coordinates }
\end{aligned}
$$




\section{COLOR RESHAPING}

The color reshaping process emphasizes the feature information of the dark area by re-adjusting the brightness value distribution of the image, and then repairs the color defects caused by the inverse HSI of the image.

$$
f_{C R_{i}}(x, y)=C_{i}(x, y) f_{i}(x, y)
$$

where $\quad f_{i}(x, y)=$ original image

$$
\begin{aligned}
& f_{C R_{i}}(x, y)=\text { image after color reshaping } \\
& C_{i}(x, y)=\text { adjustment facto } \\
& i=\text { color channel }
\end{aligned}
$$

The expression of the adjustment factor is as follows:

$$
C_{i}(x, y)=\log \frac{\kappa L_{i}(x, y)}{\sum_{j=1}^{3} L_{j}(x, y)}
$$

where $\quad \kappa=$ nonlinear strength of the adjustment factor $L_{i}(x, y)=$ Gaussian low-pass filter result.

The vividness of the color of the image after processing is improved. After the image is reshaped, the image is more natural and realistic under the human visual perception.

\section{BRIGHTNESS EQUALIZATION MODEL}

The model is mainly divided into three major steps. The first step is to adaptively adjust the brightness separated from the HSI transform. The second step is to dynamically expand the adjusted brightness and transform it into the RGB color space. The third step is to use the Gaussian blurred image as the base image to preserve the image details, and then use the color reshaped image as the top image to maintain the vividness of the image color. The processing flow is shown in Figure 2.

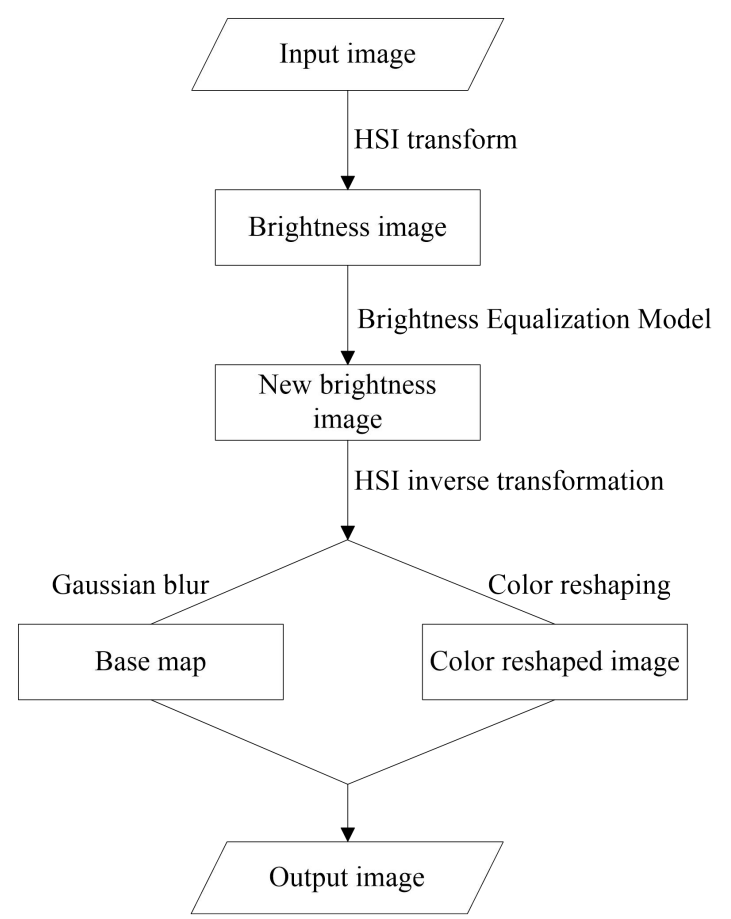

Fig.2 Flow chart of brightness equalization model

In the first step, the original image is HSI transformed according to the following transformation formula (4):

$$
\begin{aligned}
& \mathrm{r}=\frac{R}{r+g+b}, \mathrm{~g}=\frac{G}{r+g+b}, \mathrm{~b}=\frac{B}{r+g+b} \\
& h=\cos ^{-1}\left[\frac{(r-g)+(r-b)}{2 \sqrt{(r-g)^{2}+(r-b)(g-b)}}\right], h \in[0, \pi] \\
& \left.h=2 \pi-\cos ^{-1}\left[\frac{(r-g)+(r-b)}{2 \sqrt{(r-g)^{2}+(r-b)(g-b)}}\right], h \in \pi, 2 \pi\right] \\
& \mathrm{s}=1-3 \cdot \min (r, \mathrm{~g}, \mathrm{~b}), s \in[0,1] \\
& i=\frac{(R+G+B)}{3 \cdot 255}, \mathrm{i} \in[0,1] \\
& H=\frac{h \cdot 180}{\pi} ; S=s \cdot 100 ; I=i \cdot 255
\end{aligned}
$$

The separated brightness map is subjected to local filtering processing:

$$
R(i)=\sum_{j \in S u b s e t, j \neq i} \frac{r(I(i)-I(j))}{d(i, j)}
$$


The International Archives of the Photogrammetry, Remote Sensing and Spatial Information Sciences, Volume XLII-3/W10, 2020 International Conference on Geomatics in the Big Data Era (ICGBD), 15-17 November 2019, Guilin, Guangxi, China

where Subset $=$ subset template participating in the operation

$$
I(i)-I(j)=\text { the difference between the current }
$$
point $i$ and the surrounding points $j$

$$
r=\text { the relative brightness representation }
$$

function

$$
d=\text { the distance metric function }
$$

The relative brightness representation function uses a saturation function:

$$
r(x)=\left\{\begin{array}{l}
-1, x<-T \\
\frac{x}{T},-T \leq x \leq T \\
1, x>T
\end{array}\right.
$$

The distance measure function is the Manhattan distance formula:

$$
d(i, j)=\left|x_{i}-x_{j}\right|+\left|y_{i}-y_{j}\right|
$$

The second step, dynamic expansion, re-plans the brightness map and dynamically adjusts to the appropriate size [15]:

$$
O(i)=\operatorname{round}\left[127.5+\frac{127.5 \times R(i)}{M}\right]
$$

where $\quad O(i)=$ the processed luminance map

$$
M=\max [R(i)], i \in \text { Subset }
$$

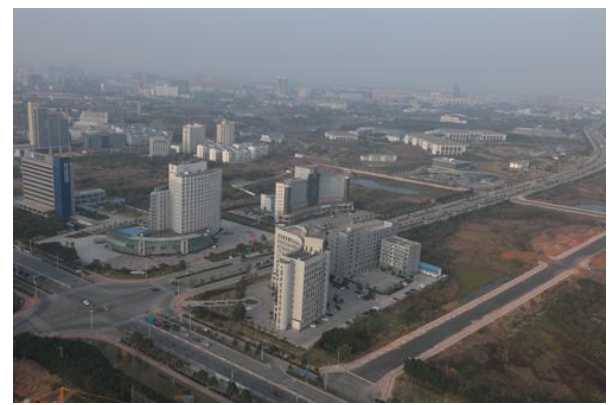

(a)Original image
The new brightness map and the original hue and saturation are inversely transformed into the RGB color space to form a new image:

$$
\begin{aligned}
& h=\frac{H \cdot \pi}{180} ; s=S / 100 ; i=I / 255 \\
& x=i \cdot(1-s) \\
& y=i \cdot\left[1+\frac{s \cdot \cos (h)}{\cos (\pi / 3-h)}\right] \\
& z=3 i-(x+y) \\
& r=y ; g=z ; b=x, h<\frac{2 \pi}{3} \\
& r=x ; g=y ; b=z, \frac{2 \pi}{3} \leq h<\frac{4 \pi}{3} \\
& r=z ; b=y ; g=x, \frac{4 \pi}{3} \leq h<2 \pi \\
& R=r \cdot 255, G=g \cdot 255, B=b \cdot 255
\end{aligned}
$$

In the third step, a Gaussian blur with a template size of $3 \times 3$ is used for $f(x, y)$ to obtain $f^{\prime}(x, y) . f^{\prime}(x, y)$ contains the texture information of the result image $f(x, y)$ of the previous step. By introducing color reshaping process, the color defects caused by the inverse HSI transformation of the image are compensated for. Finally, the image $f^{\prime}(x, y)$ is used as the base map, and $f_{C R}(x, y)$ as the top image to obtain the final result image of the brightness equalization model.

\section{COMPARISON AND ANALYSIS OF EXPERIMENTS}

In order to measure the dehazing effect of the model in this paper, the image quality evaluation criteria such as mean, standard deviation and entropy of the image were used to analyze the experimental results. Figures 3 and 4 show the results of the experiments.

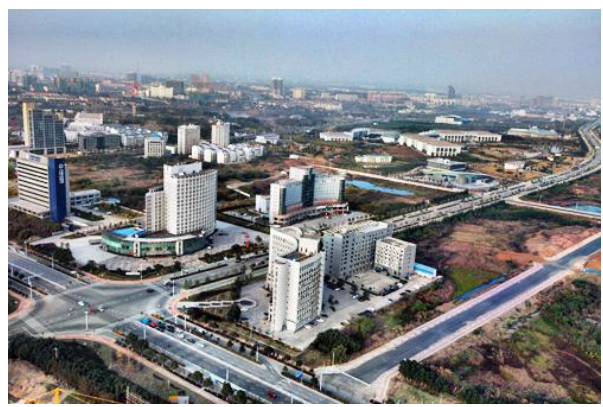

(b)The resulting image

Fig.3 Comparison of original image and processed image 
The International Archives of the Photogrammetry, Remote Sensing and Spatial Information Sciences, Volume XLII-3/W10, 2020 International Conference on Geomatics in the Big Data Era (ICGBD), 15-17 November 2019, Guilin, Guangxi, China

Table 1 Comparison of indicators of original image and processed image

\begin{tabular}{ccc}
\hline index & Original image & The resulting image \\
\hline Mean value & 107.6768 & 131.4947 \\
Standard deviation & 39.9679 & 61.6893 \\
entropy & 7.2223 & 7.8453 \\
\hline
\end{tabular}

The original image has the influence of foggy environment. The color of the image scene is dark and the change is insufficient. The brightness of the sky area and the distant building are generally white. The low contrast of the image gives a very uncomfortable feeling. The brightness equalization model of this paper emphasizes the color features of the image, and the image is obviously enhanced. The dynamic range of the color is expanded, the transition is

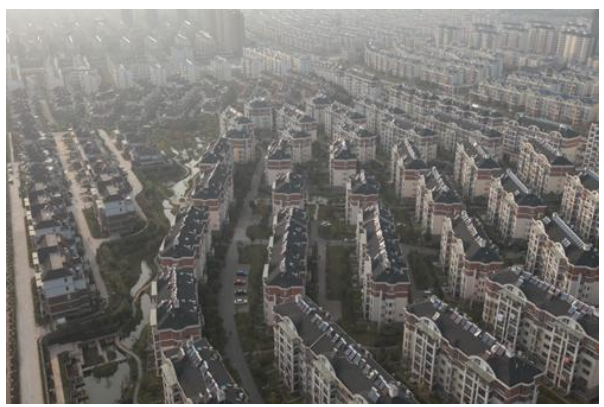

(a)Original image smooth, and the brightness can be evenly distributed. The image feature information is restored and the visual quality of the image is improved. The average value of the image is improved after processing. The increase of the standard deviation indicates that the image is easy to distinguish and the color is brighter after the image is defogged. The increase of the entropy value indicates that the information of the fogged area of the image is restored after processing.

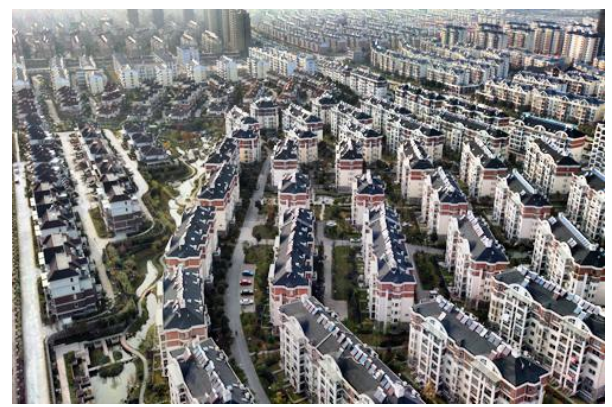

(b)The resulting image

Fig.4 Comparison of original image and processed image

Table 2 Comparison of indicators of original image and processed image

\begin{tabular}{ccc}
\hline index & Original image & The resulting image \\
\hline Mean value & 111.6438 & 121.1041 \\
Standard deviation & 42.3007 & 63.4690 \\
entropy & 7.3023 & 7.8995 \\
\hline
\end{tabular}

The original image pixels are concentrated in the middle area, and there is no difference in the brightness of the image, and the color change is not vivid enough. After being processed by the brightness equalization model in this paper, the foggy effect is significantly weakened. Similarly, the improvement of the mean and standard deviation of the image and the increase of the entropy value indicate that the image information of the image is restored after the image is processed. The contrast of the image is enhanced, and the sharpness and vividness are significantly improved.

\section{COMCLUSIONS}

Aiming at the problem of the quality degradation of the drone image due to the influence of fog, this paper proposes a brightness equalization model based on HSI transform and adaptive brightness adjustment. Through the comparison experiments, the following conclusions are obtained:

(1) The brightness equalization model uses HSI transform to obtain the brightness map of the image, and then adjusts the brightness value to the appropriate interval by adaptive brightness equalization filtering. The effect of smog in the image can be attenuated, and the visual effect of the image 
is significantly improved.

(2)In this paper, gaussian blurring processing and color reshaping processing are introduced in the brightness equalization model, which not only retains certain details but also enhances the fresh activity of color.

(3)The brightness equalization model of this paper has excellent defogging effect. And can improve the main parameters of the image, such as image brightness mean, standard deviation and entropy.The brightness equalization model of this paper has certain guiding significance and use value in the dehazing processing of images.

\section{REFERENCES}

[1] Wang Guiping, Du Jingjing, Song Jing, et al. A Fusion Filter Method for Unmanned Aerial Vehicle Remote Sensing Image Based on Gradient Inverse [J]. Science Technology and Engineering, 2018, 18(31):195-199.

[2] Zhang Li, Yang Anhong, LI Xintao, et al. Model and Realization for Island Area Images Dehazing [J]. Journal of Geomatics Science and Technology, 2010, 27(4).

[3] Tang Jianbo, Jiang Tie, Wang Tian. Research on Single Image Dehazing Using Guided Filtering [J]. Science Technology and Engineering, 2013, 13(11):3021-3025.

[4] Wang Xuemei, Ju Mingye. Fast Image Haze Removal Based on Dark Channel Prior [J]. Science Technology and Engineering, 2016, 16(20)

[5] Bi Kai, Li Yingcheng, Ding Xiaobo, et al. Aerial Photogrammetric Technology of Light Small UAV: Status and Trend of Development [J]. Bulletin of Surveying and Mapping, 2015(3)
[6] Oakley J P , Satherley B L . Improving image quality in poor visibility conditions using a physical model for contrast degradation[J]. IEEE Transactions on Image Processing, 1998, 7(2):167-179.

[7] Yu Jing, Xu Dongbin, Liao Qingmin. Image defogging:a survey[J]. Journal of Image and Graphics, 2018, 16(9):1561-1576.

[8] He K , Sun J , Tang X . Single image haze removal using dark channel prior[C]// IEEE Conference on Computer Vision \& Pattern Recognition. IEEE, 2009.

[9] Wang Shizhen, Wan Huiqiong, Zeng Lingsha, et al. Haze Removal Methods of Remote Sensing Image Using Dark Channel Prior $[\mathrm{J}]$. Journal of Geomatics Science and Technology, 2011, 28(3):182-185.

[10] Xiao Jun,Song Shoupeng,Ding Lijuan. Research on the Fast Algorithm of Spatial Homomorphic Filtering [J]. ournal of Image and Graphics,2008,13(12):2302-2306.

[11] Land E H E . Lightness and retinex theory.[J]. Journal of the Optical Society of America, 1971, 61(1):1-11.

[12] Land, Edwin H . The Retinex Theory of Color Vision[J]. Scientific American, 1977, 237(6):108-128.

[13] Fu Yiping,Li Zhineng,Yuan Ding. Improbing license plate recognition system by color algorithms based on HSI space [J]. Computer Engineering and Design,2004(05):703-707.

[14] Wang Ning,Zhang Ying. Color image edge detection based on flexible morphology of HSI space [J]. Journal of Computer Applications,2007(S2):95-96.

[15] Zhang Fanglue, Xu Xinyu, Hu Shimin. Speed optimization automatic color equalization [J]. Engineering Journal of Wuhan University, 2013, 46(6):784-789. 